Supporting Information

\title{
Rheology of Conjugated Polymers with Bulky and Flexible Side
}

\section{Chains}

Zhi-Chao Yan, ${ }^{*}, 1$ Yanan Li, ${ }^{1}$ Zhenfeng Guo, ${ }^{1}$ Akira Shinohara, ${ }^{1,2}$ Takashi Nakanishi ${ }^{1,2}$, Guangming Chen, ${ }^{* 1}$ Chengjun Pan, ${ }^{* 1}$ Florian J. Stadler, ${ }^{* 1}$

${ }^{1}$ Shenzhen Key Laboratory of Polymer Science and Technology, Guangdong Research Center for Interfacial Engineering of Functional Materials, College of Materials Science and Engineering, Shenzhen University, Shenzhen 518055, China

${ }^{2}$ International Center for Materials Nanoarchitectonics (WPI-MANA), National Institute for Materials Science (NIMS), Namiki 1-1, Tsukuba 305-0044, Japan 

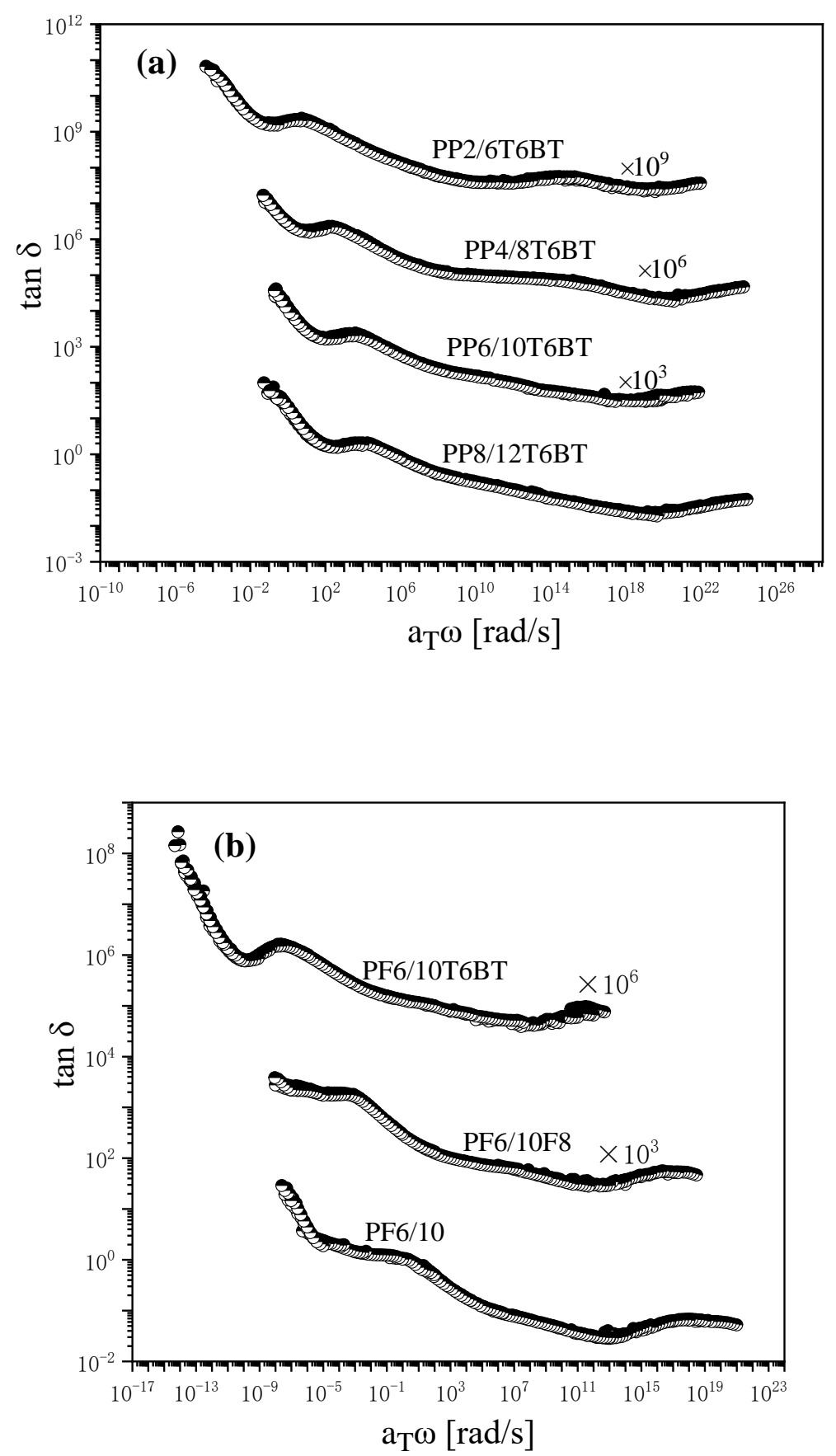

Figure S1. Loss factors ( $\tan \delta$ ) of (a) PP series: PP2/6T6BT, PP4/8T6BT, PP6/10T6BT, and PP8/12T6BT (from top to bottom) at reference temperature $T_{\text {ref }}=55^{\circ} \mathrm{C}$ and (b) PF series: PF6/10T6BT, PF6/10F8, and PF6/10 (from top to bottom) at reference temperature $T_{\text {ref }}=-10{ }^{\circ} \mathrm{C}$. Data have been vertically multiplied by multiples of 1000 , for clarity of presentation. 

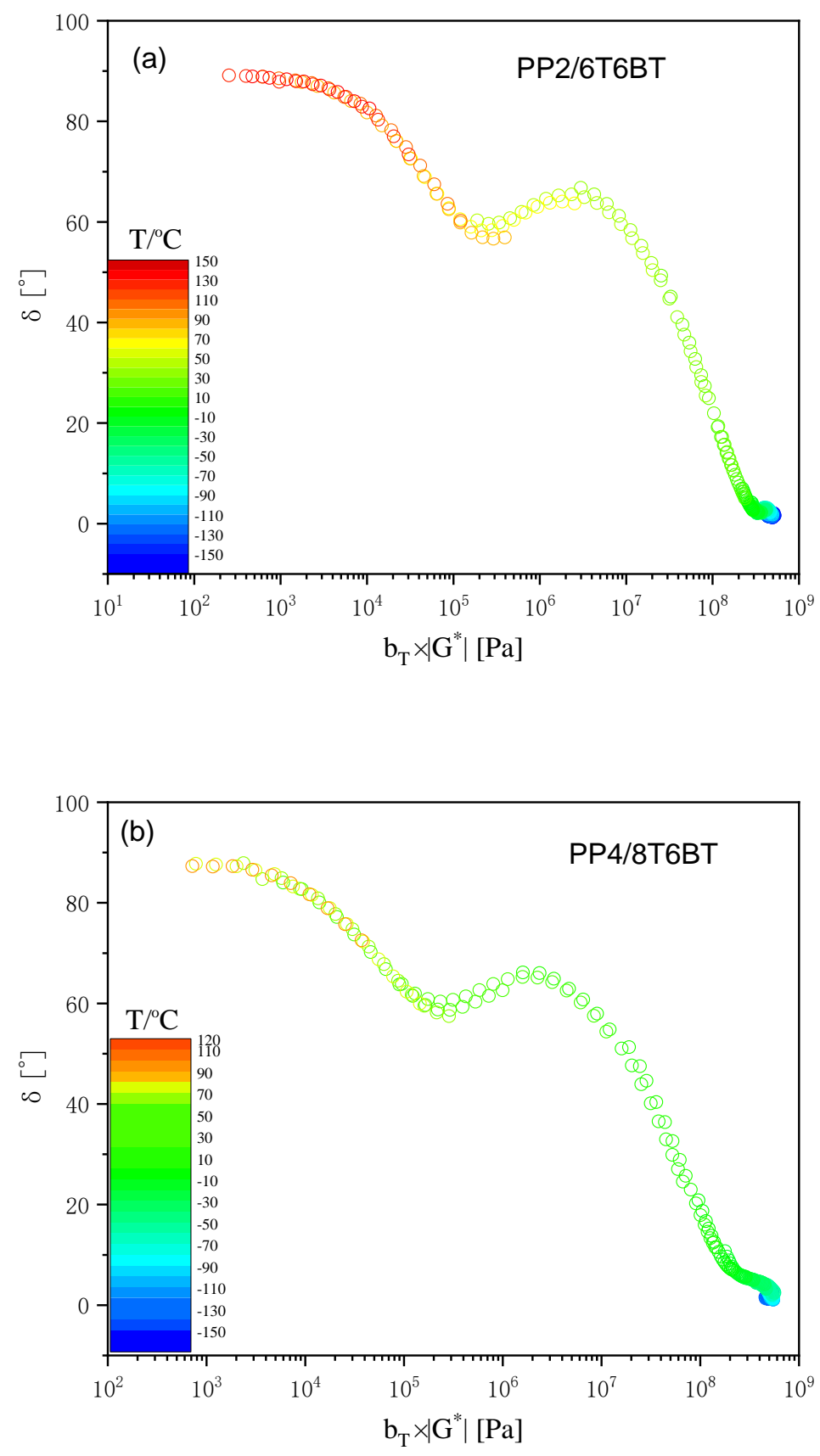

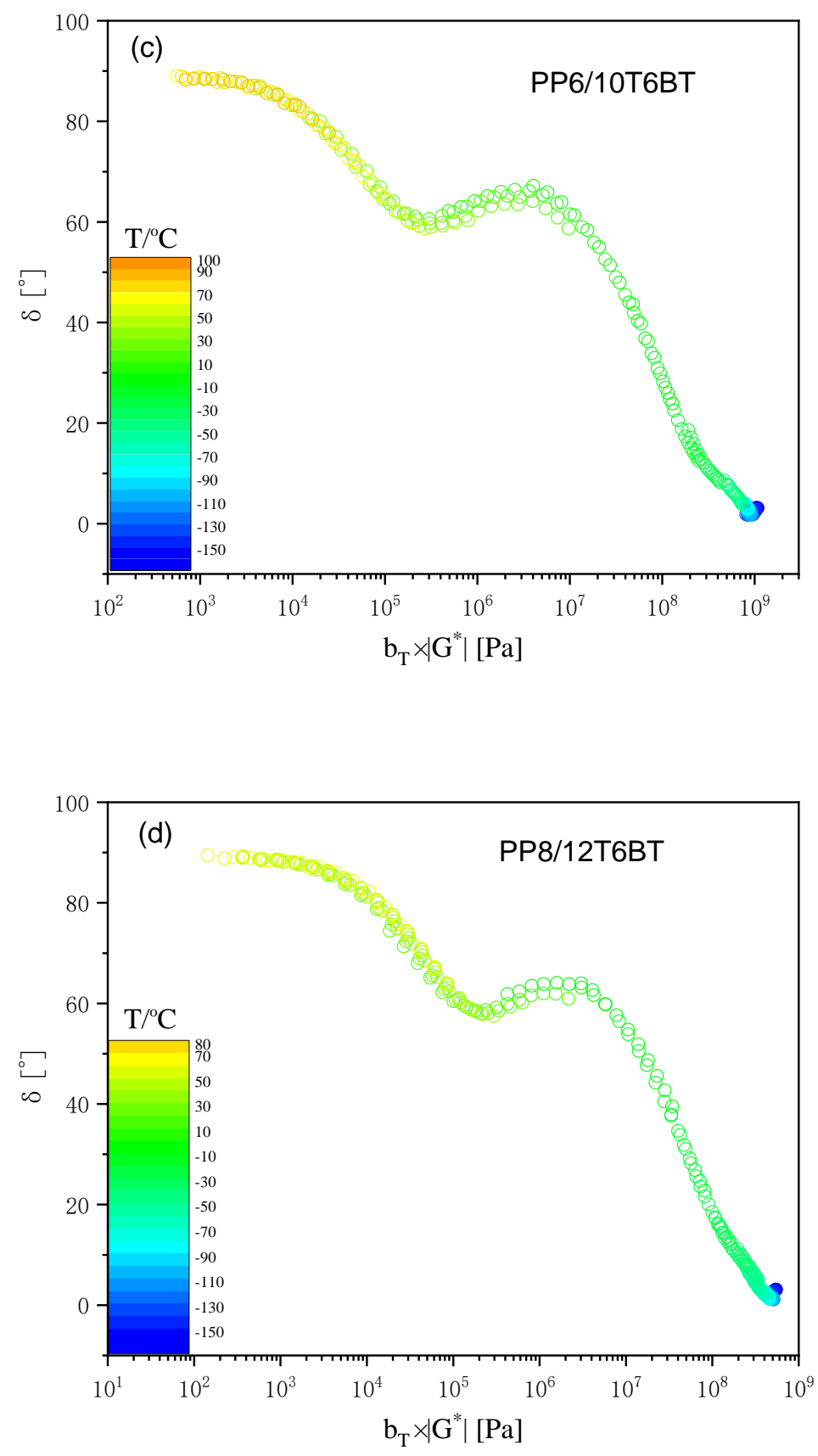

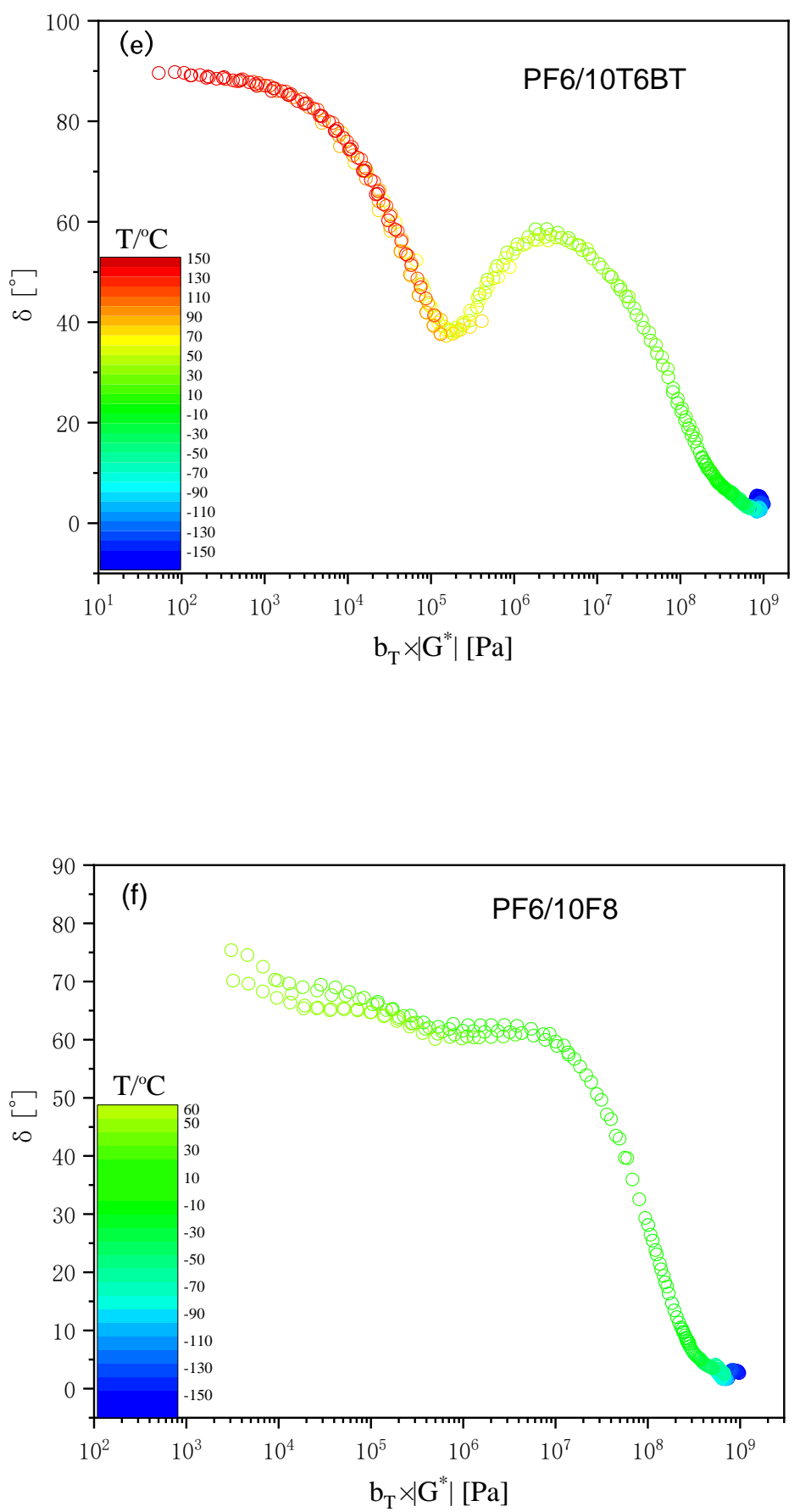


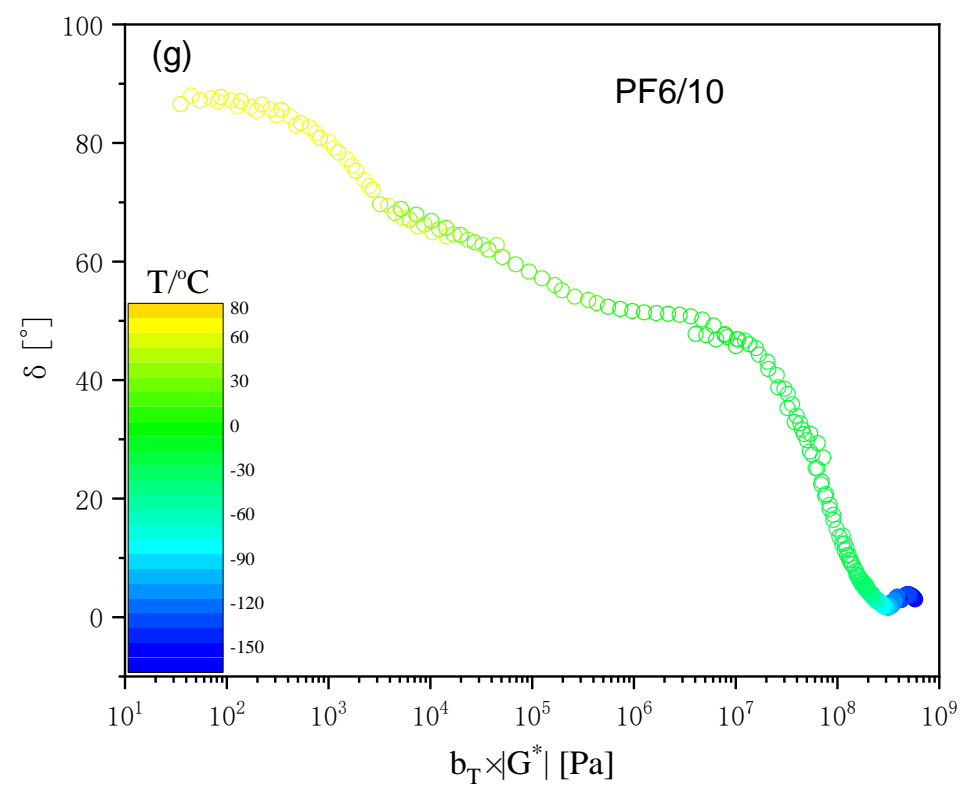

Figure S2. van Gurp-Palmen plots of (a) PP2/6T6BT, (b) PP4/8T6BT, (c) PP6/10T6BT, (d) PP8/12T6BT, (e) PF6/10T6BT, (f) PF6/10F8, and (g) PF6/10. tTS of PF6/10F8 fails at terminal regime probably due to crystalline issue. The colorbar corresponds to temperatures.

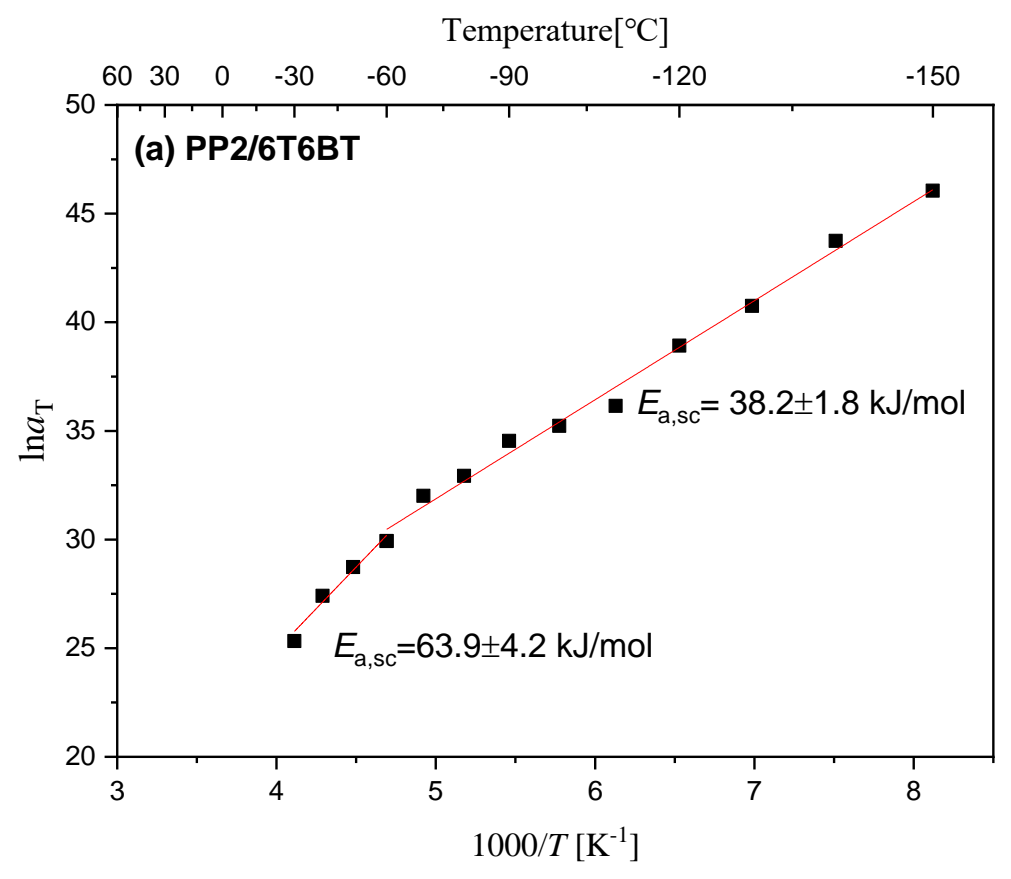



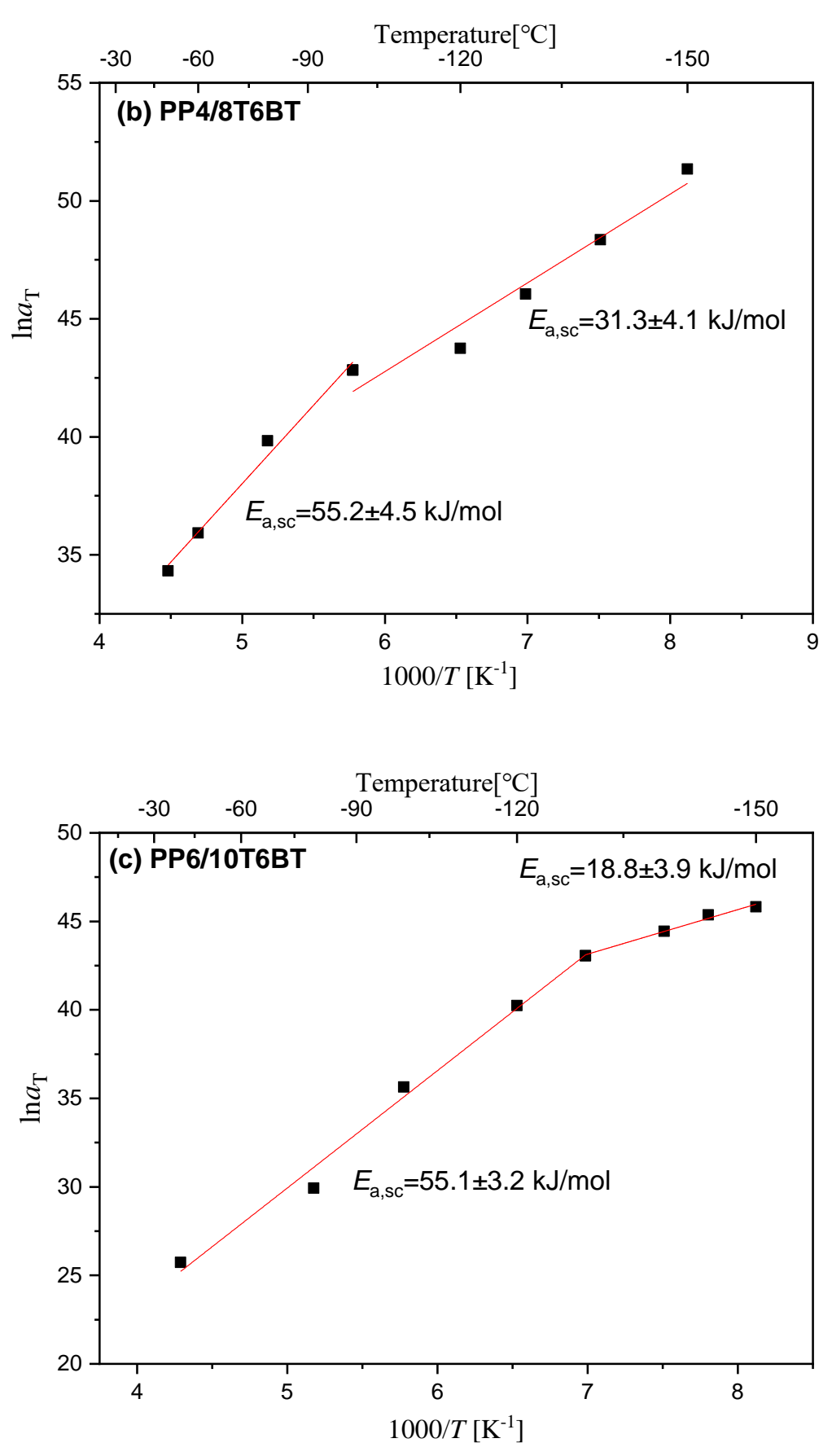

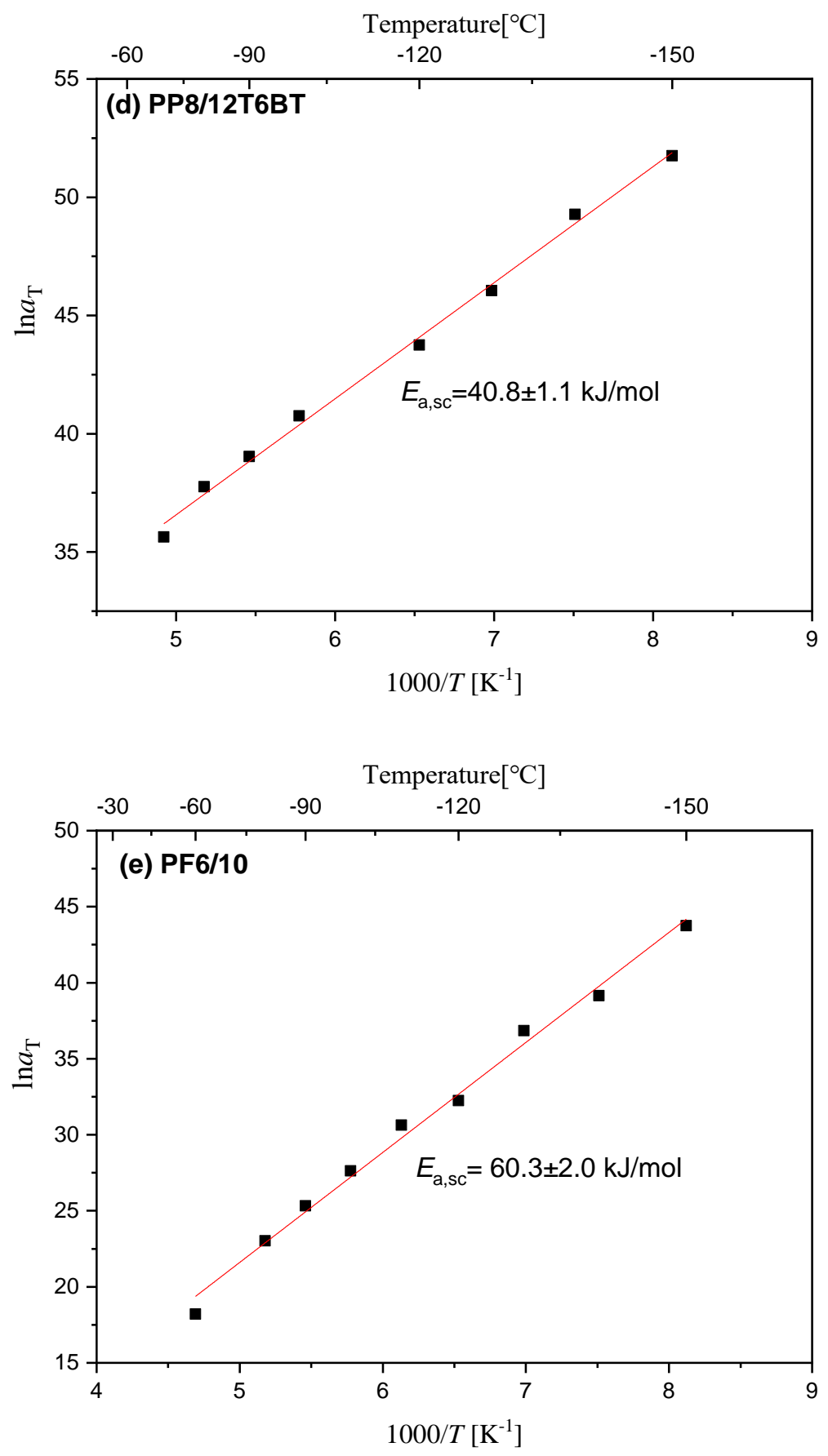


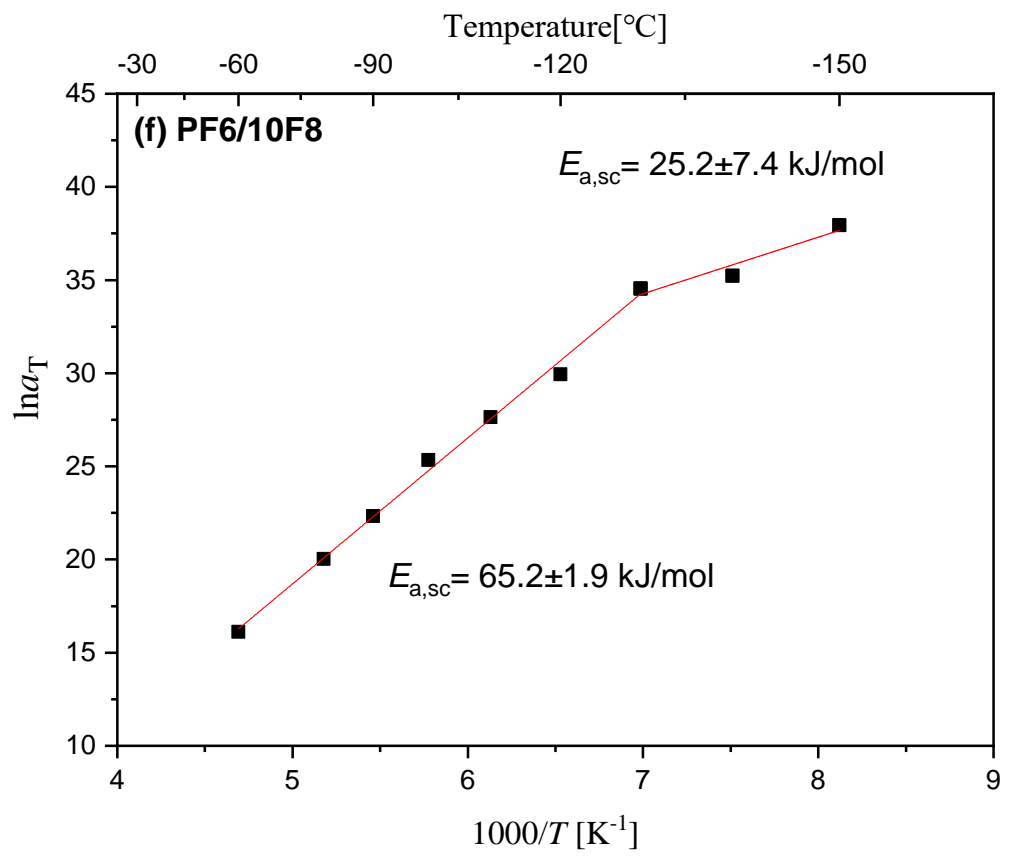

Figure S3. Arrhenius fitting to the shift factor at low temperatures for (a) PP2/6T6BT, (b) PP4/8T6BT, (c) PP6/10T6BT, (d) PP8/12T6BT, (e) PF6/10, and (f) PF6/10F8. 


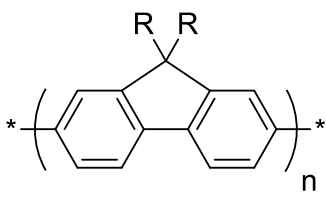

PF6: $R=n-h e x y l$

PF8: $R=$ n-octyl

PF12: $R=$ n-dodecyl

PF2/6: $R$ = 2-ethylhexyl

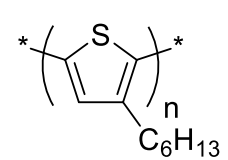

RR P3HT

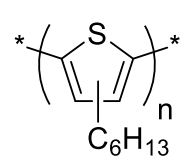

RRa P3HT

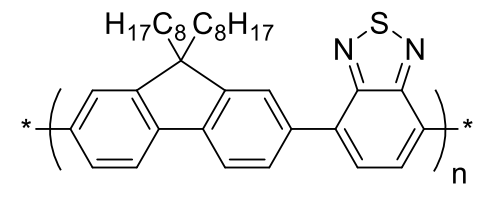

PF8BT

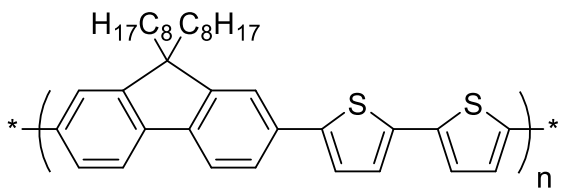

PF8T2

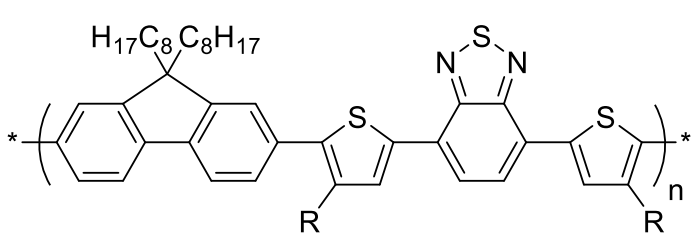

PF8TBT: $\mathrm{R}=\mathrm{H}$

PF8T6BT: $R=n$-hexyl

PF6: poly(9,9-dihexylfluorene)

PF8: $\operatorname{poly}(9,9$-dioctylfluorene)

PF12: poly(9,9-didodecylfluorene)

PF2/6: poly[9,9-bis(2-ethylhexyl)fluorene]

P3HT: poly(3-hexylthiophene) (RR: regioregular; RRa: regiorandom)

PF8BT: $\operatorname{poly}(9,9$-dioctylfluorene-alt-2,1,3-benzothiadiazole)

PF8T2: poly(9,9-dioctylfluorene-alt-bithiophene)

PF8TBT: poly(9,9-dioctylfluorene-alt-4,7-dithieno-2,1,3-benzothiadiazole)

PF8T6BT: poly-(9,9-dioctylfluorene-alt-4,7-bis(4-hexyl-2-thienyl)-2,1,3-

benzothiadiazole).

Scheme S1. Chemical structures of selected polymers from ref 1

\section{References:}

1. Xie, R.; Weisen, A. R.; Lee, Y.; Aplan, M. A.; Fenton, A. M.; Masucci, A. E.; Kempe, F.; Sommer, M.; Pester, C. W.; Colby, R. H.; Gomez, E. D. Glass Transition Temperature from the Chemical Structure of Conjugated Polymers. Nat. Commun. 2020, $11,893$. 